\title{
Les guides touristiques : vers de nouvelles pratiques discursives de contamination
}

Annabelle Seoane

\section{(2) OpenEdition}

12 Journals

Édition électronique

URL : http://journals.openedition.org/tourisme/81

DOI : 10.4000/tourisme.81

ISSN : 2492-7503

Éditeur

Éditions touristiques européennes

\section{Édition imprimée}

Date de publication : 1 décembre 2013

Pagination : $33-43$

ISSN : 2109-5671

\section{Référence électronique}

Annabelle Seoane, «Les guides touristiques : vers de nouvelles pratiques discursives de contamination », Mondes du Tourisme [En ligne], 8 | 2013, mis en ligne le 01 juin 2015, consulté le 10 décembre 2020. URL : http://journals.openedition.org/tourisme/81 ; DOI : https://doi.org/10.4000/ tourisme.81

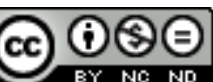

Mondes du tourisme est mis à disposition selon les termes de la licence Creative Commons Attribution - Pas d'Utilisation Commerciale - Pas de Modification 4.0 International. 


\title{
Les guides touristiques : vers de nouvelles pratiques discursives de contamination
}

\author{
ANNABELLE SEOANE_[annabelleseoane@yahoofr] \\ Docteur en sciences du langage, CREM, Université de Lorraine
}

Résumé. C'est selon la démarche de l'école française d'analyse du discours que nous abordons le discours des guides touristiques français comme une production discursive inscrite dans un champ social, mais qui tend à s'ouvrir à d'autres pratiques discursives par un phénomène de contamination. Nous nous interrogeons sur la manière dont des pratiques communicationnelles extra-discursives (internet, presse, télévision) influent directement sur des pratiques discursives en étant des facteurs d'évolution; nous montrerons que les pratiques discursives sont de moins en moins étanches entre les différents médias : le contenu informatif et la routine, caractéristiques du guide touristique, ont tendance à s'exporter vers d'autres genres discursifs (multimédia, presse papier) qui, à leur tour, l'influencent et le modèlent selon leurs propres normes. Qu'elle s'effectue par le type d'énonciation des nouveaux médias ou par celui des magazines, la contamination est la résultante d'une adaptation des discours des guides touristiques aux pratiques sociales. Ces discours s'inscrivent dès lors dans un champ social qui leur est à la fois spécifique (le discours à teneur touristique) et général (les discours de transmission de savoirs), s'appuyant parfois sur des communautés discursives qu'ils contribuent eux-mêmes à établir.

Abstract. Following the traditional French school of discourse analysis, the discourse of French guidebooks is approached as a discursive production registered in a social field, which tends to open up to other discursive practices through a contamination phenomenon. We examine how some extra-discursive communication practices (Internet, written press and television) directly affect discursive practices by playing the role of evolution factors; we show how discursive practices are less and less hermetic to other kinds of media. The information carried and the routine specific to guidebooks tend to shift to other discursive genres (multimedia or written press) which will in turn influence and shape them according to their own standards. Whether it is carried out by the enunciation types of new media or by magazines, the contamination is the result of an adaptation of the discourse of guidebooks to social practices. These discourses fit into a social field, which is both specific (discourses with touristic content) and general (knowledge transmission discourses), sometimes relying on discourse communities, which they contribute themselves to establish. 
$\mathbf{P}$ ar une approche dans la tradition de l'école française d'analyse du discours, nous aborderons le discours des guides touristiques français comme une production discursive inscrite dans un champ social, telle que souvent définie par Catherine Kerbrat-Orecchioni ou Dominique Maingueneau, entre autres. Comme nous l'avons fait précédemment dans des travaux issus de recherche doctorale (Seoane, 2013), nous avons choisi de travailler sur un corpus constitué de guides des plus connus, emblématiques de leur époque et que leurs destinations ancrent dans une situation extra-culturelle (le Cambodge) ou en situation intraculturelle (la Bretagne). Ces situations interculturelles placent le lecteur dans un univers inconnu (ou méconnu) dans lequel nombre de ses repères s'estompent. Le guide, dans son discours didactique, se doit alors à la fois de rendre compte, de faire une sélection, de donner envie et de rassurer. Partant d'un savoir partagé, il crée un nouveau cadre. Le guide, en tant que voyageur, ethnologue et pédagogue, voit et fait voir le référent nouveau (un lieu, le plus souvent) à travers le prisme de sa culture et de ses connaissances. Nous utiliserons également, avec une visée contrastive, des guides plus anciens, comme le Guide bleu (Monmarché, 1924) ou le Guide Thiolier (1934) sur la Bretagne.

Quel que soit le guide, une sorte de cahier des charges commun semble apparaître. Le discours des guides touristiques est mû par une vocation commerciale (il faut se vendre pour perdurer) et une vocation didactique (conseiller des itinéraires, décrire des monuments et des sites, dépeindre la faune et la flore, sélectionner le couvert ou le gîte...). Un guide est tenu de fournir, dans un souci de clarté et de lisibilité, une information qui se donne pour objective (autant qu'elle puisse l'être) sur les lieux de destination et sur les conditions de voyage, d'accueil et de séjour. Sa mission est à la fois de préparer et d'accompagner le lecteur dans son séjour, ce qui suppose en amont une prise en compte des diverses formes de tourisme possibles, élaborée à partir d'un profil type de la clientèle, ciblée selon ses desiderata et ses attentes. Il doit ensuite définir la spécificité culturelle du référent : au-delà de l'identité qui est la sienne va s'opérer la nécessité de reconstruire une nouvelle identité stéréotypique, issue de l'imaginaire collectif du lectorat visé (un public français avide de dépaysement, par exemple). Le but n'est pas tant de décrire une contrée que de créer discursivement un ailleurs désirable et accessible, concrétisé par le voyage effectif du lecteur. En valorisant et en mettant en discours un certain espace touristique, le guide s'inscrit dans une époque et dans une culture donnée. Les professionnels de la communication touristique parlent alors de "mise en tourisme" de la destination.

Le guide s’ingénie ainsi à allier les fonctions mathésique (transmission des savoirs) et mimésique (des- cription et présentation (Adam, 1992)) à une fonction communicative à visée commerciale, cette dernière étant loin d'être secondaire ; il suit un cahier des charges sensiblement identique et dont il finit par émaner une certaine routine.

Aujourd'hui, ce discours se tourne vers de nouvelles pratiques discursives de contamination pour s'assurer un écho maximal auprès du public. En effet, l'information à caractère touristique est semblable à un bien de consommation, omniprésente et pluricanale : elle se retrouve traditionnellement dans les guides, mais également, depuis quelques années internet, dans la presse et les chaînes télévisuelles. Le lecteur-touriste change de statut au fil de l'essor de ces contenus informatifs touristiques : lecteurtéléspectateur ou, sur internet, lecteur-internaute, il consomme autant qu'il dispense aisément des informations qu'il désigne "conseils", "tuyaux" ou "bons plans". Ainsi, dans les médias passifs (TV, internet web 1.0) et dans les médias plus participatifs (le web 2.0), le lecteur a accès à un éventail extrêmement large d'informations qu'il peut, dans ce dernier cas, contribuer directement à enrichir encore.

Ce flux informatif continu, destiné essentiellement à l'accompagner dans la préparation de son séjour autant qu'in situ, remplit un contrat pragmatique analogue à ceux du guide touristique, mais avec un effet décuplé puisque hors des limites matérielles du format papier ; le destinataire ne dispose plus simple- 
ment d'un choix d'une dizaine d'hébergements proposés par le guide mais de milliers sur le web. Avec quelles conséquences sur les pratiques discursives? Le locuteur se construit-il un positionnement dans son discours (l'ethos) adapté à ces nouvelles pratiques de consommation ? Autrement dit, comment des pratiques extra-discursives influentelles directement sur des pratiques discursives en étant des facteurs d'évolution?

Nous verrons que les pratiques sociétales de consommation ont induit de nouvelles pratiques discursives, et les fonctions jusque-là réservées au guide touristique se sont disséminées à d'autres supports. Parallèlement, ce phénomène de "dissémination" s'est doublé d'un phénomène de "contamination", par lequel ces nouveaux supports ont influencé la mise en discours dans les guides touristiques. Nous montrerons que ces pratiques ont conduit à une dissémination du dit autant qu'à une contamination des manières de dire.

Pour finir, nous verrons dans quelle mesure ces pratiques s'ancrent dans des communautés discursives, qu'elles contribuent elles-mêmes à créer d'ailleurs, et donc, par ce biais, comment elles s'inscrivent dans le champ social. Cela nous permettra de montrer dans quelle mesure la porosité aux pratiques communicationnelles et discursives est le principe même de l'évolution des guides touristiques depuis leur apparition. Les pratiques discursives sont de moins en moins étanches entre les différents médias : le contenu informatif et la routine caractéristiques du guide touristique ont tendance à s'exporter vers d'autres genres discursifs (multimédias ou la presse papier) qui vont à leur tour l'influencer et le modeler selon leurs propres normes.

\section{STABILITÉ OU HYBRIDATION ?}

Bien qu'ils aient actualisé leur format et leur contenu au fil des années, le Guide du routard ou le Guide bleu déploient tous deux un ethos (c'est-à-dire une image de soi construite dans et par son discours) stable et très identifié, qui a peu évolué depuis leur création, ce qui en a favorisé la popularité et a sans doute permis dès lors le maintien d'une stabilité scénographique ${ }^{(1)}$ dans le temps.

Au contraire, d'autres guides tels que le Guide Gallimard, le Petit Futé, le Lonely Planet ou le Guide vert, par exemple, ont des identités scénographiques sans doute moins marquées, moins reconnues donc, et ils forment alors des pratiques communicationnelles moins stabilisées (richesse de l'iconographie, hyperstructure de la page, tonalité adoptée...). L'objectif communicatif du locuteur doit répondre également aux contraintes du marché concurrentiel : en fonction de la production éditoriale pour une destination, par exemple, les guides cherchent un positionnement qui leur permettrait de se démarquer là où, justement, le foisonnement des discours touristiques favoriserait plutôt une cer- taine homogénéisation des pratiques, comme pour le Guide Gallimard qui varie de scénographie selon qu'il se place en situation intra ou extra-culturelle (Seoane, 20I3). À cet égard, la dissémination des savoirs à d'autres supports a contribué à rendre ces pratiques discursives de moins en moins étanches par un phénomène de contamination.

Étudions à présent la contamination par les médias, nouveaux (internet) ou exogènes (la presse magazine).

\section{CONTAMINATION}

\section{PAR LES NOUVEAUX MÉDIAS : ERGONOMIE DU DISCOURS}

Dans son article intitulé "Le mélange des genres dans l'hyperstructure", Gilles Lugrin a désigné cette "rencontre de deux médias, à savoir la presse et internet", comme une "hybridation" (Lugrin, 200 I, p. 90). La contamination par les nouveaux médias correspond aux nouveaux modes de consommation de l'information, pour lesquels "l'aspect ergonomique” est prépondérant, et au sujet desquels Lugrin a parlé de "design d'information" et d' "esthétisation" du contenu informatif (200I, pp. 93-94).

L'information tend à devenir fragmentée et la lecture parcellaire, selon le modèle calqué sur les hyperstructures d'internet et autres nouvelles technologies de l'information.

En ce qui nous concerne, nous parlerons plus volontiers de "contamination" que d' "hybridation", car 
il s'agit davantage d'une influence de la discursivité du web que vraiment d'un mélange de discours. Cependant, nous garderons à l'esprit la démarche de Gilles Lugrin (200 I) ou de Jacques Mouriquand (1997), entre autres, et émettons l'hypothèse que le discours touristique a subi une évolution similaire à celle du discours journalistique dans la reconstruction de sa structure d'ensemble pour accroître sa "vi-lisibilité” (Seoane, 20I3). Cette évolution favorise l'apparition de nouveaux modes d'organisation textuelle.

Dans le discours journalistique, pour reprendre les catégories définies par Lugrin (200I, pp. 88-92), les principales conséquences en sont :

- la réduction de la longueur moyenne des descriptions : l'information est rendue plus synthétique ; - le rubriquage plus marqué, qui encourage une lecture "mosaïque" et non linéaire (et le phénomène de "zapping") et qui "se pense comme une somme non séquentielle d'informations, somme qui rend possible de multiples parcours de consultation" (Lugrin, 200 I, p. 9I); - la circulation du sens est mise en avant à l'intérieur de l'hyperstructure grâce à une information plus facilement repérable, qui "revêt une forme d'immédiateté, une lecture presque simultanée de l'information” (Lugrin, 200 I, p. 88);

- la mise en spectacle de l'information : le développement du visuel et de la mise en page par une iconographie et une infographie plus présentes et plus riches;

- une interdiscursivité( ${ }^{(2)}$ par le biais de formes textuelles "commentatrices" (de l'ordre du métadiscursif parfois) qui alimentent le contenu informatif. "À ces 'exigences de visibilité' et 'de lisibilité' s'ajoute 'l'exigence d'intelligibilité' qui concerne le commentaire qui est fait sur l'événement. Elle se manifeste surtout dans les formes textuelles 'commentatrices' (éditoriaux, chroniques, analyses, etc.)" (Lugrin, 2001, p. 91).

Pour le discours des guides touristiques, notre démarche partira du postulat selon lequel, historiquement, il a existé deux types de guides : les guides dits "littéraires" et les "pratiques". Nous utiliserons le critère du visuel pour montrer que leur évolution n'a pas suivi la même trajectoire, ce qui nous permettra de cerner le phénomène de contamination par les discours des nouveaux médias.

\section{Les guides "littéraires"}

Le plus emblématique des guides “littéraires”, le Guide bleu, semble avoir conservé son identité scénographique (ethos, mise en page) jusque dans les années 2000. Les scans du Guide bleu Bretagne de 1924 (Monmarché, 1924) montrent une mise en page épurée, quasi sans illustrations, un texte longuement développé (six pleines pages pour Vannes, par exemple), sans mise en colonnes ni séquençage très marqué, une maquette peu aérée ( $c f$. figure 1).

À la suite du Guide bleu sont ensuite apparues d'autres collections, comme celles du Guide Gallimard ou du Guide vert : l'hyperstructure y est renforcée par une titraille mise en exergue, des espaces plus nombreux et une mise en pages sur deux colonnes, les photographies en couleur font leur apparition, les sites incontournables sont particulièrement mis en valeur. L'objectif didactique est atteint par le visuel autant que par le contenu. Cette mutation se trouve renforcée par le marché concurrentiel de l'édition touristique. En effet, pour le Cambodge, les quelques guides existants se positionnent comme des guides "pratiques", comme une réponse de terrain à la situation extra-culturelle : le Routard (Gloaguen, 2004, 2007), le Lonely Planet, le Petit Futé essentiellement. À cet égard, le Guide Gallimard se positionne souvent dans un registre plus "littéraire", avec les caractéristiques discursives qui s'ensuivent (hyperstructure moins segmentée, style plus littéraire, recours à l'hypotaxe, choix lexicaux plus soutenus, etc.). En revanche, pour la Bretagne, de très nombreux guides coexistent, c'est pourquoi, dans ce contexte, la concurrence favorise la contamination puisque le Guide Gallimard semble opter pour un positionnement différent et devient alors plus perméable à la contamination par les nouveaux médias. Ainsi, pour la Bretagne, il se définit lui-même comme "Encyclopédie du voyage" (Collectif, 2007), ce qui correspond à l'accumulation de textes courts, fragmentés et à forte visée didactique, alors que pour le Cambodge, comme "Bibliothèque du voyageur", ce qui 
renvoie à une dimension plus littéraire et esthétisante (Fève et Milledrogues, 200 I) (cf. figure 2).

Si nous comparons le traitement du fait culturel dans ces deux guides, cette dichotomie est clairement visible. L'hyperstructure de ces parties caractérise la volonté d'institution d'un cadre communicationnel différant selon le locuteur.

\section{Les guides dits "pratiques"}

Le Guide Thiolier, dans les années 1930, faisait déjà montre d'une volonté d'arquer son discours sur le visuel. Outre la concision des descriptions, les photographies, les infographies (schémas, cartes, itinéraires), les encadrés publicitaires en marge du discours principal témoignent déjà d'un “mélange des genres" dans l'hyperstructure, bien avant l'apparition des discours multimédias ( $c f$. figure 3).

Dans ce guide de 1934, le souci didactique occupait une place moindre que la vocation commerciale. Le contenu informatif était donné par un discours monologique et non “embrayé", largement contrebalancé par un fort dialogisme - interlocutif: "Non, Mesdames!! la gourmandise n'est plus un défaut" (p. 6), ou interdiscursif: "Guides Thiolier, moyen de diffusion unique" (p. 3) - et des discours annexes, hétéroclites, présentés sous forme d'encadrés et donc structurellement disjoints. Ces encadrés aux contenus souvent "embrayés" témoignaient de l'importance du moment de l'énonciation (le contexte historico-sociologique de l'époque) dans le discours touristique, et de la facilité pour celui-ci à assimiler les discours environnants.

Ces discours périphériques (auto-désignés comme "bas de pages") s'ancrent dans le "faire savoir" et le "faire faire" caractéristiques des discours touristiques, mais à un niveau plus universel, macroénonciatif ; ils sont le lien entre le locuteur qui recommande et le lecteur, le moins ciblé possible: "Nos bas de pages. À nos lecteurs, quelle que soit leur situation, millionnaires ou Français moyens, nous recommandons nos bas de pages pour les méditer, en tirer profit, les mettre en pratique pour le bien de tous. Merci!” (p. 26).

Le locuteur assoit son autorité le plus souvent par des arguments factuels, chiffrés : "550 000 : tel est le tirage officiel des Guides Thiolier [...] Publicité la moins chère et la plus appréciée [...] Consultez les pages vertes" (p. 2) ; "Ne voyagez pas sans un Guide Thiolier, toute la France pittoresque en 18 régions" (p. 8) ; "95\% des Guides Thiolier touchent la clientèle touristique qui sait voyager et apprécier le Beau et le Bon” (p. 25). Parfois, les arguments avancés témoignent d'un

\section{Figure | • Guide bleu Bretagne (1924)}
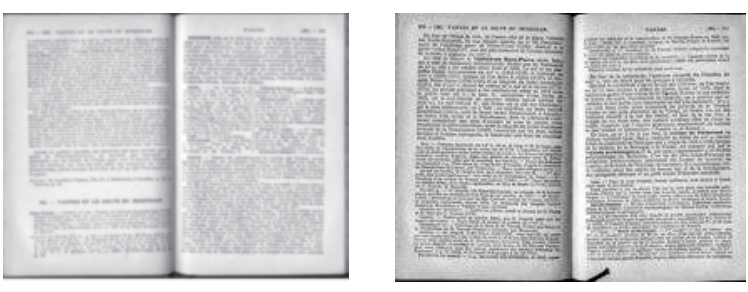

Figure 2 • Guides Gallimard

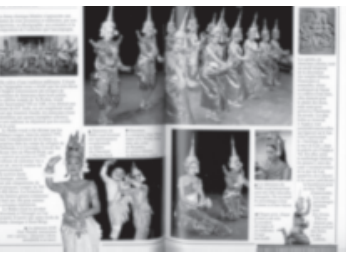

Cambodge (200I)

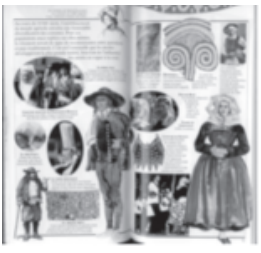

Bretagne (2007)
Figure 3 • Guides Thiolier (1934)

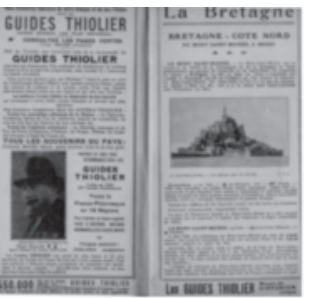

effort pour créer une connivence avec le lecteur par l'autodérision : "Les Guides Thiolier évitent les coups de fusil et servent d'armure" (p. 18).

Le locuteur justifie son autorité également par sa relation à cette forme de transcendance de l'énonciation : "Comme les Guides Thiolier, les syndicats d'initiative favorisent le développement $d u$ tourisme en France et nous font connaître à l'étranger. Aidez-les généreusement!" (page de couverture). 
Une petite digression historique nous permettra de souligner l'évolution de l'ethos du locuteur-guide : dans les années 1930, dans le sillage de la crise de 1929 aux États-Unis, la France connaît un ralentissement de son activité économique, aggravé par la chute de son commerce extérieur déjà déficitaire. Apparaît alors une tendance marquée au protectionnisme et à la promotion du terroir français. Le locuteur se fonde donc sur ce contexte socio-historique pour développer un ethos de guide patriotique et moderne. Il exacerbe un sentiment nationaliste où le tourisme devient un outil stratégique :

"Les Guides Thiolier font aimer et connaître la France, apprécier les plats régionaux, les produits culinaires, crus et liqueurs” (p. 40); "Pour gagner la bataille touristique, un but unique; la vraie, la bonne cuisine française, nos vins feront le reste..." (p. 4l).

Ce positionnement lui permet d'asserter des énoncés sur les deux plans d'énonciation, ce qui contribue à stabiliser son autorité discursive et montre la forte intrication des pratiques extra-discursives du moment et le contenu du discours du Guide Thiollier.

- Premier exemple. Parallèlement à ce contexte de crise économique, la pratique de la publicité se développe ; les annonces commerciales permettent d'abaisser le prix des parutions, d'en étendre le lectorat et d'en optimiser la rentabilité : " $L a$ Publicité, la Concurrence et l'Initiative sont l'âme du commerce" (p. 32) ; "La Publicité est une puissance moderne qui décuple la valeur d'une entreprise bien menée” (p. 33).

- Deuxième exemple. Au début du $\mathrm{XX}^{\mathrm{e}}$ siècle, le tabac devient un produit de grande consommation; là encore, le locuteur tire parti de son environnement pour adapter son discours et faire passer des messages à caractère sociétal qui sortent totalement du champ touristique: " $\mathrm{Ne}$ fumez pas en mangeant. La cuisine française ne supporte pas le goût du tabac... et vous gênez vos voisins" (p. 45).

En lien direct et explicite avec son contexte, le locuteur ne se donne pas simplement comme guide touristique mais presque comme guide à penser et du savoir-vivre, ce qui renforce son autorité discursive autant que son impact sur le lecteur.

Ce retour sur le passé dans les guides touristiques va nous permettre d'affiner notre analyse sur la contamination par les discours des nouveaux médias. La perméabilité du Guide Thiolier aux discours et aux pratiques de son époque montre bien que ce sont les mêmes mécanismes d'adaptation aux cadres de communication qui sont en œuvre aujourd'hui par les phénomènes de contamination. En revanche, le Guide bleu tel qu'il se conçoit historiquement s'est montré extrêmement peu poreux aux discours environnants, si bien qu'il a continué d'exister sous sa forme initiale, mais son éditeur a dû développer d'autres collections qui, elles, ont pu donner lieu à la mise en discours des nouvelles pratiques touristiques.

Ainsi, le phénomène de contamination n'est pas nouveau et il est au centre même de la question du genre. La circulation du sens et des discours, qui repose sur la diminution de la longueur moyenne des descriptions, le développement du visuel, la lecture "mosaïque", la "mise en spectacle" de l'information et l'interdiscursité sont autant d'éléments qui permettent aux guides de rester en adéquation avec les attentes de leur public. La multitude des supports et des formats médiatiques présents de nos jours pose la question de l'avenir du guide sous sa forme "Guide bleu", tant l'interférence est prégnante avec les discours environnants. Le format presse magazine en est le plus marquant.

\section{CONTAMINATION}

\section{PAR LA PRESSE MAGAZINE}

Le chapitre consacré à la gastronomie dans chaque Guide Gallimard s'avère particulièrement révélateur de cette interférence entre le discours des guides touristiques et le discours très visuel de la presse magazine, notamment à travers ces trois entrées: d'abord, la présence de recettes de cuisine, puis la présentation d'un pêle-mêle de spécialités locales qui rappelle les pages “shopping” des magazines, et, enfin, des photographies qui manifestent une recherche esthétique du pittoresque proche de celle des magazines. 
Figure 4 - Recettes de cuisine

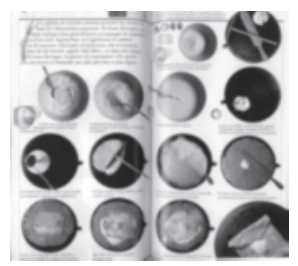

Guide Gallimard

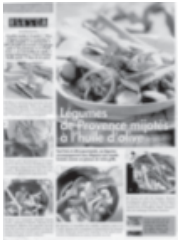

Femme actuelle

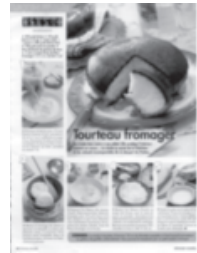

Guide Gallimard
Figure 5

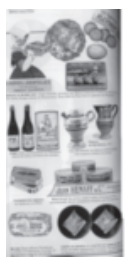

Elle

Figure $6 \bullet$ Pages shopping

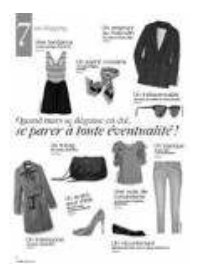

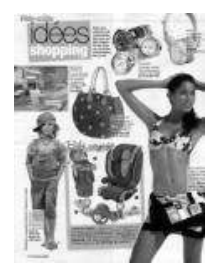

Femme actuelle
Le Guide Gallimard Bretagne présente des recettes qui se veulent prototypiques de la cuisine locale (par exemple la galette au sarrasin pour la Bretagne ou le bœuf au gingembre pour le Cambodge) et s'inscrit dans le cadre de l'objet intrinsèquement culturel, en accord avec la doxa, définie comme "l'opinion dominante sur ce qui se pratique dans un contexte donné" - "la doxa est un point de vue particulier, le point de vue des dominants, qui se présente et s'impose comme point de vue universel”, selon Pierre Bourdieu (1994, p. 129). Nous allons montrer que la doxa surplombante de la "tradition" - à savoir l'opinion partagée par tous, le sens commun - dresse un premier parallèle avec la doxa présente implicitement dans le discours des magazines. Par "doxa”, les analystes du discours entendent plus précisément "l'ensemble des croyances et opinions partagées qui sous-tendent la communication et rendent l'interaction possible" (Charaudeau et Maingueneau, 2002, p. 547).

La présence d'un texte de recette étape par étape tisse de prime abord un lien avec les fiches recettes que l'on peut trouver dans plusieurs magazines féminins généralistes, notamment dans la rubrique "Leçon filmée" ( $c f$. figure 4) du magazine Femme actuelle, magazine le plus lu de la presse féminine payante (selon l'Office de justification de la diffusion).

Dans la "leçon filmée" de Femme actuelle, les gestes ou les étapes illustrés sont banals ("Pelez les carottes", "Faites cuire les asperges", "Ajoutez le bouillon de volaille”) ; ils ne présentent pas de difficultés techniques et ne nécessitent pas d'outils inhabituels qui pourraient justifier les photographies, mais les illustrations mettent en exergue l'objectif didactique de cette "leçon" et produisent un effet de "direct live", pour reprendre une expression du jargon télévisuel comme nous invite à le faire l'épithète "filmée" : le discours simule le déroulement de l'action devant les yeux du lecteur qui devient alors virtuellement (télé)spectateur. Le Guide Gallimard reprend donc cette mise en scène d'un magazine à succès et souligne par l'image l'effet d' “exotisation" produit par l'utilisation de la galetière.

Cette recette est complétée par une sorte de pêle-mêle de "spécialités" qui, là encore, reprend les codes des maquettes de la presse magazine. À l'instar de la composition des pages "mode" ou "shopping" des magazines, plusieurs textes très courts légendent les photographies des spécialités culinaires bretonnes ( $c f$. figure 5).

Quoique la démarche ne soit pas procédurale comme dans la recette, ce texte associe également discours verbal et iconographique :

- la combinaison description-visualisation crée des "représentations mentales" qui favorisent le dépaysement (la "vi-lisibilité" d'Adam, 1992, § 70). La dimension narrative s'efface au profit d'une dimension descriptive ;

- la présence de l'énonciateur est marquée par cette segmentation de l'énoncé avec, sous-jacente, la force illocutoire du conseil.

La description de l'offre touristique renvoie explicitement au contrat générique que le locuteur honore par des procédés déjà présents dans la recette : des reformulations explicatives ou descriptives, un repérage temporel ou spatial (la désignation de l'origine des produits sert de gage de qualité aussi bien que d'évocation d'une association produit-lieu supposée connue par le lecteur). 
L'association d'éléments iconographiques et verbaux, dans la recette d'un plat stéréotypique ou dans un "pêle-mêle" de spécialités locales, met à la portée de tout lecteur une part d'exotisme, mais un exotisme "aménagé", un dépaysement préparé et sous contrôle. Pour le locuteur-guide, la recette peut, à cet égard, être considérée comme une sorte de mise en abîme de l'interaction entre le verbal et le monde extra-verbal dans lequel il se projette : un ailleurs exotique et rassurant, virtuellement à la portée de tous.

Malgré une volonté d'effacement énonciatif, la présence du locuteur est marquée par cette segmentation du dit. Il se dégage donc, en filigrane, un ethos de locuteur qui allie une précision des connaissances, un positionnement en retrait mais efficace avec un souci constant de concret, et qui fonde sa recherche de nouveauté ou d'exotisme sur des présupposés communs parfois stéréotypiques.

Comparons à présent brièvement ce type de discours mosaïque à celui que l'on peut trouver dans les pages consacrées au shopping dans les magazines féminins, par exemple (cf. figure 6, Elle et Femme actuelle de mars 2010). Nous distinguerons trois caractéristiques principales. 1. L'hyperstructure est analogue : l'éclatement de l'information est renforcé par une iconographie riche, plus ou moins esthétisante selon le locuteur.

2. L'intentionnalité didactique se retrouve dans les deux supports : même si le discours du Guide
Gallimard opte pour l'effacement énonciatif du locuteur, contrairement à ceux des deux magazines, les objets présentés sont décrits selon des approches similaires :

- des reformulations explicatives ou descriptives ("une robe marinière", "style gavroche - nouvelle longueur pour la salopette”) ;

- un repérage spatial à travers le nom de l'enseigne qui les diffuse ("Gérard Darel”, "Vert Baudet”); - un marquage social (réel ou rêvé) à travers la mention du prix.

3. Le fondement de la description sur un univers de référence partagé, avec un fort ancrage socio-culturel : les "lunettes Ray-Ban" sont considérées comme "un indispensable", le "trench" comme "un intemporel", le "jean slim" comme "un basique".

D'un point de vue pragmatique, dans le guide comme dans le magazine, le lecteur reconnaît cet univers de référence ; il est d'abord mis en confiance puis incité à participer lui-même à son propre dépaysement. De lecteur, il devient potentiellement acteur par le biais des "représentations mentales" qui lui sont fournies. Cela conduit à une primauté de l'énonciatif sur l'informatif, comme le formule Sophie Moirand (2007, p. 74) concernant l'analyse du discours de la presse quotidienne : "Ce qui compte, c'est peut-être moins ce qui est dit que le fait de le dire aux destinataires du média."

Dans les deux cas, il s'agit moins d'informer que de montrer que le locuteur se situe dans cette dynamique didactique de support pour le lecteur et de l'ancrer par ce biais dans un contexte socio-culturel tacite.

Si le Guide Gallimard met volontiers en évidence le pittoresque dans "la recette du bœuf au gingembre", "la recette de la galette au blé noir", la mosaïque de "spécialités" bretonnes et les photographies d'autochtones cambodgiens, c'est pour y exacerber la couleur locale. Le guide table sur une véritable mise en scène de ce pittoresque : celui d'un ailleurs exotique en situation extra-culturelle ou celui d'un ailleurs dit "gastronomique" en situation intra-culturelle. Cependant, à ce qualificatif de "gastronomique" sont apposées des "spécialités" comme les crêpes dentelle, les sablés, le cidre, le pâté Henaff, le beurre salé qui s'apparentent plus résolument à des mets populaires. Un double décalage transparaît donc ici dans le guide : d'une part, entre les routines du genre touristique et le genre de la recette de cuisine qui le rapproche des magazines, et, d'autre part, entre la volonté de "gastronomie" et le contenu populaire des "spécialités".

Comme l'a expliqué Roland Barthes à propos des recettes données par les magazines Elle et L'Express: "C'est parce que Elle s'adresse à un public vraiment populaire qu'elle prend bien soin de ne pas postuler une cuisine économique. Voyez L'Express, au contraire, dont le public exclusivement bourgeois est doté d'un pouvoir d'achat confortable: sa cuisine est réelle, non magique; Elle donne 
la recette des perdreaux-fantaisie, L'Express, celle de la salade niçoise. Le public de Elle n'a droit qu'à la fable; à celui de L'Express on peut proposer des plats réels, assuré qu'il pourra les confectionner" (Barthes, 1957, p. 121).

Selon cette théorie, le Gallimard proposerait des plats "réels", dont la faisabilité ne fait aucun doute (y compris pour la recette asiatique), aux ingrédients simples et au discours procédural très didactisé. Cependant, l'utilisation insistante d'ustensiles locaux (le "rouable", par exemple) en limite la réalisation. À cet égard, son public s'enracinerait donc dans une identité bourgeoise autant avérée que rêvée, comme en témoigne la présence de mets populaires.

Le Guide Gallimard met en scène le pittoresque en se donnant non seulement comme guide touristique in situ mais comme album de voyage où les saveurs du voyage peuvent se retrouver (contrat scénographique) : saveur d'un ailleurs gastronomique en Bretagne, saveur d'un ailleurs exotique au Cambodge, par exemple. Cette dichotomie est le fruit de la représentation que le lecteur est supposé se faire de son voyage et des attentes qu'il est censé avoir. Ce jeu sur la culture représentée (représentée dans le texte par le locuteur, et représentée en amont par le lecteur) n'a de sens que si le lecteur correspond à l'image de lui que s'est construite le locuteur, son lecteur modèle. Si le lecteur est breton ou cambodgien, par exemple, l'équilibre entre ces représentations mutuelles est rompu et la scénographie dysfonctionne (rien de plus banal que la galette de sarrasin ou le bœuf cuisiné au gingembre pour l'autochtone...). Cette même interaction entre les représentations mutuelles se retrouve dans le discours des magazines : le lecteur d'Elle et celui de Femme actuelle n'ont pas les mêmes attentes, c'est pourquoi la gamme de prix des produits diffère en conséquence. Elle, notamment, se positionne dans une identité qui tient du fantasme pour le lecteur par l'évocation de produits présentés comme des prototypes d'une certaine catégorie sociale :

"Un it-bag. Sac Nina Ricci. $2200 €$. [...] Un réconfortant. Ballerines Dior. New Look en agneau perforé noir. $380 €$. [...] Un basique. Un jean slim. 7 for All Mankin. $240 €$.”

C'est cette prise en compte des représentations du lecteur et de leurs interférences avec celles que projette le locuteur qui favorise la contamination du discours touristique du Guide Gallimard par le discours des magazines, comme c'est le cas également par le biais de l'iconographie.

\section{L' ICONOGRAPHIE}

\section{COMME DÉCORUM}

\section{DANS LE GUIDE GALLIMARD}

La richesse iconographique est un autre aspect de la contamination du discours de certains guides par les magazines. En effet, cette abondance de photographies dépasse le cadre de la visée d'information du

\section{Figure 7 • Guide Gallimard}
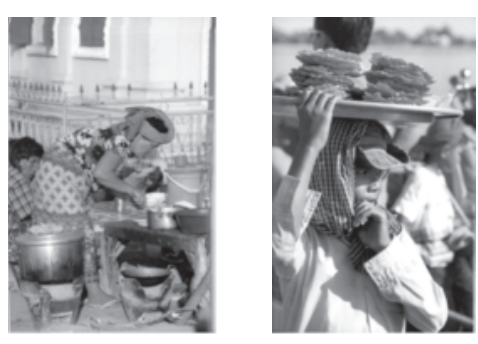

guide, elle vise davantage à renforcer la représentation (ethos) que donne le locuteur de lui-même : humaniste, esthète... Ainsi, reprenons le référent de la gastronomie dans le Guide Gallimard (Fève et Milledrogues, 200 I, pp. 232 et 237), la partie "La cuisine cambodgienne" s'ouvre et s'achève sur deux photographies pleine page qui nous semblent dignes d'intérêt par leur taille, et leur position dans le discours : cette démarche nous permet de dégager des "passerelles" avec les discours présents dans la presse-magazine ( $c f$. figure 7 ).

Même si, sans doute, ces photographies ont été prises sur le vif et avec spontanéité, leur cadrage puis leur sélection à cet emplacement précis sont le fruit d'une réflexion scénographique qui n'est pas sans dévoiler la présence d'un émetteur "scénariste" visant à rendre une réalité vue comme exotique. Cet exotisme est en partie de son fait, car le locuteur est garant de l'énonciation touristique et des contraintes génériques que celle-ci suppose, mais il est surtout du fait de son lecteur, qui s'attend à trouver ce type de scène (tant sur place que dans son guide). "La recherche de 
dépaysement n'est ici que le symptôme d'une nécessité inscrite au coeur du médium" (Fresnault-Deruelle, 1993, p. 204).

Le "médium" crée aussi bien qu'il véhicule cette attente de dépaysement. Il y a donc un fondement de nature stéréotypique à chacune de ces deux images. La pose des sujets participe également de cette recherche scénographique. Le lecteur-touriste assiste comme spectateur à ces deux scènes, sans y prendre part. Le regard de chaque personnage photographié se tourne vers la droite, vers le hors-champ. Le premier est de profil, le deuxième de trois-quarts, ce qui "permet an personnage d'être à la fois dans le récit [...] et dans le discours [...]. Le monde réel (celui d'où je regarde cette image) s'est trouvé un instant anéanti, et remplacé dans le même temps par cet autre monde où a opéré la projection. Ce trois-quarts, le regard droit n'est pas l'indice d'une recherche de contact [mais d'] une posture $d u$ discours" (Fresnault-Deruelle, 1993, p. 26).

L'absence de sourire et de jeu de regards avec le lecteur/spectateur marque que l'objectif ici n'est pas tant de communiquer directement avec le destinataire mais de le transformer en tiers, plus passif. Celuici n'est pas un "tu", mais un "il" pour qui se déploie le dispositif énonciatif de l'image.

Le réel s'ancre dans le moment du dire, puisqu'il montre des sujets “dans le récit”, en pleine action dans un environnement dans lequel ils s'intègrent (spatialement, chro- matiquement). Cette narrativité donnée par le cadrage (impression de "prise sur le vif"), la pose, les couleurs rendent compte de façon indirecte d'une prise de position d'un locuteur vers le lecteur (jeu de regards vers le hors-champ).

La relation énonciative apparaît dans un rapport au réel biaisé par les représentations stéréotypiques (sujets, couleurs) de cette réalité vue comme exotique et donnée comme pittoresque, où le hors-champ accentue l'effet d'évasion. C'est la raison pour laquelle, dans cette partie consacrée à la "gastronomie cambodgienne", l'objet n'est pas tant la gastronomie en tant que telle mais l'environnement de cette gastronomie, son décorum en quelque sorte, qui contribue au dépaysement requis par le contrat scénographique du guide touristique et que le locuteur "scénariste" tâche de remplir pour le lecteur "spectateur". C'est une passerelle entre le discours des guides et le discours des magazines (de presse, de voyages, féminins, etc.).

\section{LA CONTAMINATION}

COMME FACTEUR D'ÉVOLUTION

Qu'elle s'effectue par le type d'énonciation des nouveaux médias ou par celui des magazines, la contamination est la résultante d'une adaptation des discours des guides touristiques aux pratiques sociales. Ces discours s'inscrivent dès lors dans un champ social qui leur est à la fois spécifique (le discours à teneur touristique) et général (les discours de transmission de savoirs). À cet égard, l'imbrication de types d'énonciations exogènes différentes révèle des positionnements propres à chacun dans ce champ social. Les notions de "communauté discursive" et de "communauté de parole" sont déterminantes pour montrer l'interaction entre le verbal et les processus sociaux.

Selon nous, les guides touristiques utilisent ainsi des processus sociaux dans lesquels les énoncés produits sont en accord avec des normes collectivement reconnues et attendues. Insérées dans le champ des discours à visée didactique, ces normes s'inscrivent dans une volonté de constitution d'un réseau, une communauté discursive, sur lequel elles fondent leur légitimité et qui leur assurent une certaine porosité à l'égard de l'interdiscours.

Du point de vue commercial, cette porosité répond à une nécessité cruciale de s'adapter aux nouveaux enjeux de la société. Du point de vue énonciatif, elle répond à un double mouvement issu de pratiques sociales nouvelles : un phénomène de dissémination des fonctions du guide touristique dans d'autres supports de communication (presse magazine, chaînes télévisuelles, internet, smartphones...) et un phénomène concomitant de contamination des contenus des guides par les discours environnants (ceux des magazines, par exemple).

\footnotetext{
NOTES

(I) Le concept de "scénographie" a été défini par Maingueneau (Le Contexte de
} 
l'œuvre littéraire. Énonciation, écrivain et société, Dunod, 1993) : "Dans une scénographie s'associent une figure d'énonciateur et une figure corrélative de co-énonciateurs. Ces deux places supposent également une chronographie (un moment) et une topographie (un lieu) dont pretend surgir le discours. [...] La scénographie, pour jouer pleinement son rôle, ne doit donc pas être un simple cadre, un décor, comme si le discours survenait à l'intérieur d'un espace déjà construit et indépendant de ce discours : l'énonciation en se développant s'efforce de mettre progressivement en place son propre dispositif de parole. Elle implique ainsi un processus en boucle. Dès son émergence, la parole suppose une certaine situation d'énonciation, laquelle, en fait, se valide progressivement à travers cette énonciation même. La scénographie est ainsi à la fois ce dont vient le discours et ce qu'engendre ce discours ; elle légitime un énoncé qui, en retour, doit la légitimer, doit établir que cette scénographie dont vient la parole est précisément la scénographie requise" (Charaudeau et Maingueneau, 2002, p. 516).

(2) Tout discours est traversé par l'interdiscursivité, car il a pour propriété constitutive d'être en relation avec d'autres discours, réalisés souvent antérieurement et avec lesquels se constitue une sorte de "dialogue", par un jeu de renvois internes.

\section{RÉFÉRENCES BIBLIOGRAPHIQUES}

Jean-Michel ADAM, Les Textes : types et prototypes, coll. "Fac. Linguistique", Nathan, 1992.

Roland BarTHes, Mythologies, coll. "Points", Seuil, 1957.

Pierre BOURDIEU, Raisons pratiques. Sur la théorie de l'action, coll. “Points", Le Seuil, 1994.

Patrick Charaudeau et Dominique Maingueneau, Dictionnaire d'analyse du discours, Seuil, 2002.

Collectif, La Bretagne, Guides Thiolier, 1934.

Collectif, Bretagne, coll. "Encyclopédies du voyage”, Gallimard, 2007.

Nathalie Fève et Lucie Milledrogues, Le Grand Guide du Laos et du Cambodge, coll. "Bibliothèque du voyageur", Gallimard, 200 I.

Pierre Fresnault-Deruelle, L'Éloquence des images. Images fixes III, coll. "Sociologie d'aujourd'hui", Puf, 1993.

Philippe Gloaguen (dir.), Le Guide du routard : Cambodge, Laos, Hachette Tourisme, 2004.

Philippe Gloaguen (dir.), Le Guide du routard : Bretagne Sud, Hachette Tourisme, 2007.

Gilles LUGRIN, "Le mélange des genres dans l'hyperstructure", Semen, n 13 : "Genres de la presse écrite et analyse de discours", 200 I [http://semen.revues.org//635].

Sophie MolRand, Les Discours de la presse quotidienne, Puf, 2007. Marcel MonMARCHÉ (dir.), Bretagne, Guides bleus, Hachette, 1924. Jacques MOURIQUAND, L'Écriture journalistique, coll. "Que sais-je ?", Puf, 1997.

Annabelle SEOANE, Les Mécanismes énonciatifs dans les guides touristiques : entre genre et positionnements discursifs, L'Harmattan, 2013. 